\title{
Applications of X-ray Micro Computed Tomography Technology in Food Research
}

\author{
Abigail D. Lape ${ }^{1}$, Var St. Jeor ${ }^{1}$, Janice Johnson ${ }^{2}$ and Paul Smith ${ }^{3}$
}

${ }^{1}$ Cargill, Research \& Development - Ingredient, Material, and Nutrition (IMN), Plymouth, MN USA.

2. Cargill, Salt - Food Applications and Technical Service, Excelsior MN, USA.

3. Cargill, Research \& Development - Ingredient, Material, and Nutrition (IMN), Vilvoorde, BE.

Traditionally, x-ray micro CT (XRMCT) systems have been used in the medical field or academic research. Cargill is a food company that applies this technology to fully characterize our internal and external customer's products. X-ray tomography is an analytical tool that uses the density differences of components to create a 3-dimensional rendering of the object. As the sample is rotated 360 degrees, an image projection is collected at incremental angles. These images are reconstructed to form a composite 3-D image which can be viewed at any angle, optically sliced in any plane and analyzed for quantitative information. This is done in a non-destructive manner with little or no sample preparation preventing the introduction of false or misleading artifacts. The collected images and information gives the ability to view internal structure, can be used to assess product quality, and is useful in guiding future experiment/prototype design. X-ray tomography in combination with other analytical tools provides a distinctive capability in Cargill's research toolbox and is well aligned with the building of the core technology area of structural design and material science.

XRMCT can often be utilized in qualitative analysis or in monitoring product quality. The nondestructive aspect of this technique enables the visualization of a product without physical alteration. In the quality control area, defects in the product including delamination, internal cracking, or bubble formation can be easily identified (Figure 1A). The density differences in the material can also be used to discover foreign material including high density metals or to visualize ingredient distribution (Figure 1B). Additionally, the reconstructed images enables a scientist to quickly measure coating thickness and coverage in products (Figure 1C). Another advantageous aspect of this technique is the ability understand the effects of process changes such as freeze/thaw cycles (Figure 1D).

XRMCT technology is also used to fully characterize new or existing products. As an example, FlakeSelect ${ }^{\mathrm{TM}}$ is an innovative low sodium solution that leverages Cargill's patent-pending compacting technology to combine and agglomerate ingredients. The overall sodium content is reduced by producing a salt that is composed of both of sodium and potassium chloride. The use of scanning electron microscopy (SEM) and energy dispersive spectroscopy (EDS) confirmed the presence of both elements on the surface of the particle. XRMCT's 3D capability was utilized to digitally slice through the particle and visualize the presence of each element throughout the entire particle. The combination of complementary analytical techniques confirmed the composition and stability of this new product (Figure 2).

Fundamental food research benefits from the use XRMCT to understand ingredient behaviour and the ability to connect this functionality within a system. This is extremely relevant in the food industry where there is a call for healthier or clean label choices along with a push for reduction in fat, salt, and sugar. The removal or substitution of these ingredients often affects the microstructure translating into a change in sensory perception and customer liking of the product. One area of interest is the reduction of oil uptake in food products. An XRMCT scan of fried instant noodles visualizes the internal structure 
and potential pathway for liquid oil to enter the system. The number of pores and their interconnectedness can affect the amount of oil that is taken up. The reconstructed image of a noodle can now be analyzed for porosity, oil content, and total void volume. The oil can be segmented to measure the relative amount and its distribution within the noodle. The proximity of oil to a pore can give insight to the mechanism of how it enters into the system (Figure 3). This study of microstructure and oil behaviour gives direction to potential additives or processing changes that can reduce the porous structure of the noodle.

X-ray micro computed tomography can be used in many areas of food research and development. It is a versatile tool that can be used to fully characterize products, understand effects of process and ingredient changes, a guide to direct next steps in product development, and a capability applied in quality control.

A
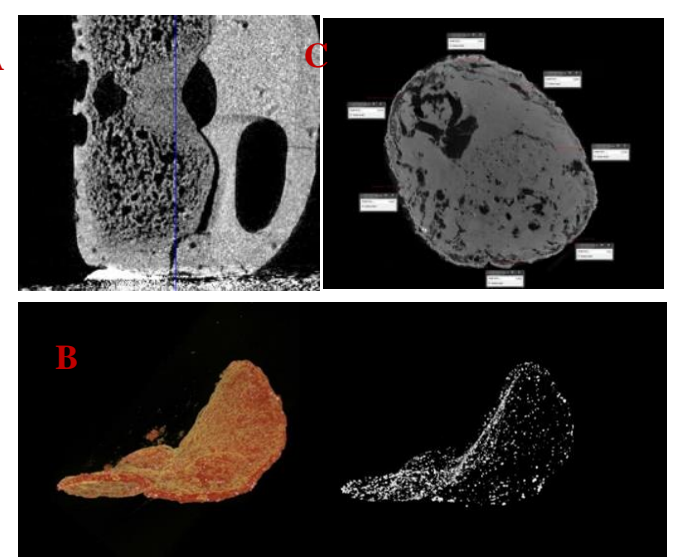

Figure 1. A-D. Use of XRMCT as a qualitative tool or in quality control. A) Delamination, internal cracking and bubble formation in a candy bar. B) Salt distribution on a potato chip. C) Visualization and quick measurement of coating coverage in a chicken patty. D) CT scan of icing before and after freezing.
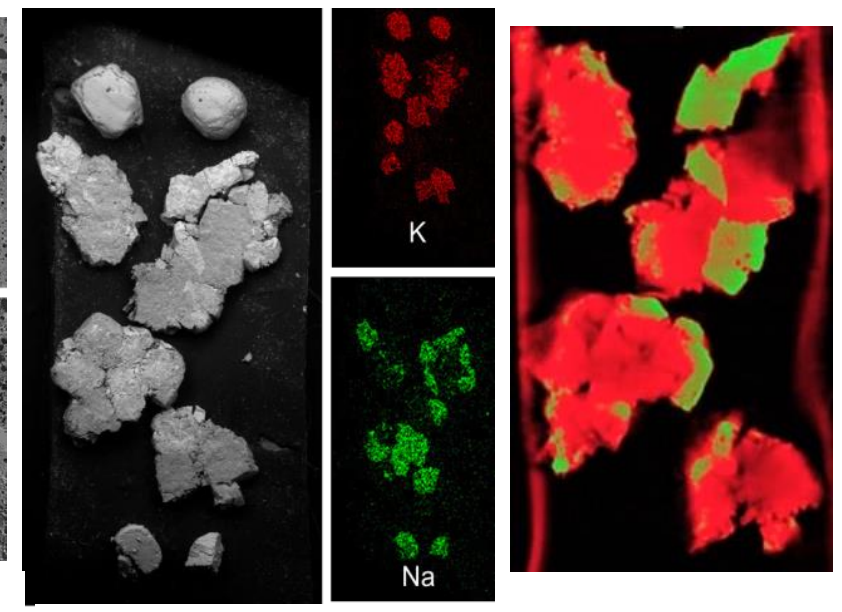

Figure 2. FlakeSelect ${ }^{\mathrm{TM}}$ product characterization using scanning electron microscopy, energy dispersive spectroscopy and x-ray MicroCT.
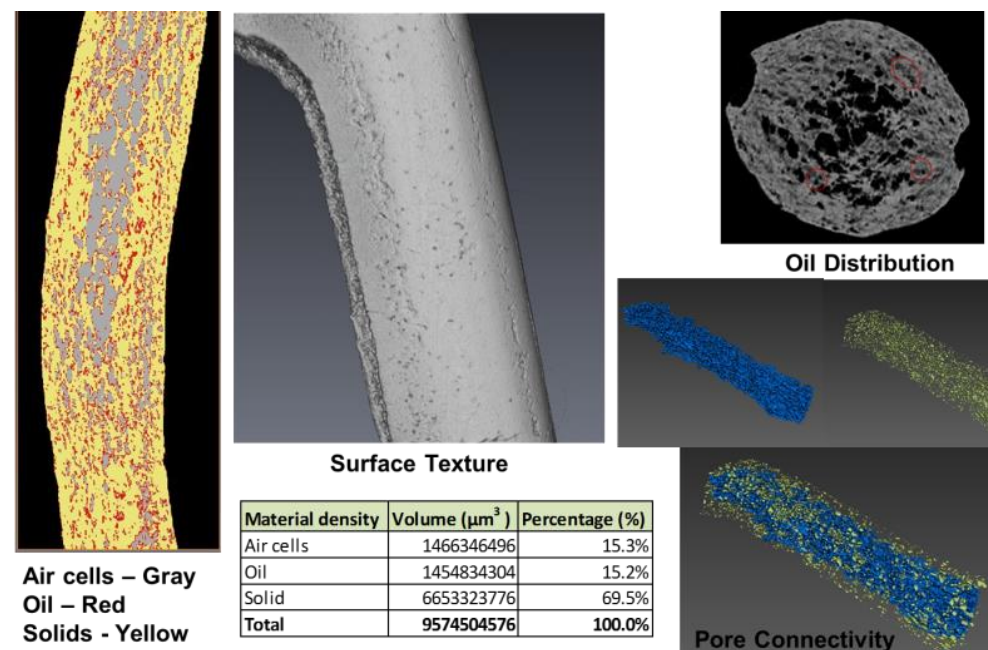

Oil Distribution

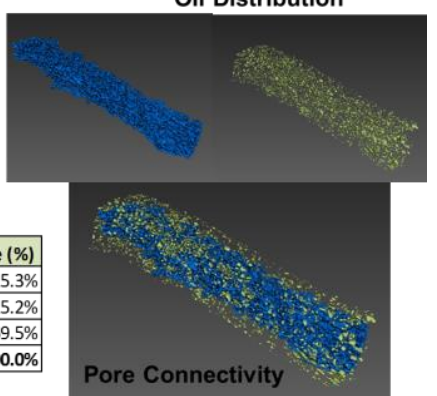

Figure 3. XRMCT is used to visualize the surface texture, oil distribution, and pore connectivity to understand pathways for oil uptake. 